the right to post a revised personal version of the text of the final journal article (to reflect changes made in the peer review process) on the author's personal or institutional web site or server, incorporating the complete citation and with a link to the Digital Object Identifier (DOI) of the article;

Article title: Brain regions concerned with perceptual skills in tennis: an fMRI study

Reference: INTPSY9646

Journal title: International Journal of Psychophysiology

Corresponding author: Dr. M.J. Wright First author: Dr. M.J. Wright

Received at Elsevier: 21-APR-2006 DOI information:

$10.1016 / j$.ijpsycho.2006.03.018

This is a pre-puffication version.

Final manuscript Pubfistied as:

Wright, Migand Lackson, KG. (200y). Brain regions concerned with perceptuas skifs in tennis: an filgh fstudy. International Journal of Psychophysiology $63214-220$ 


\title{
Brain regions concerned with perceptual skills in tennis: an fMRI study
}

\author{
Michael J. Wright ${ }^{* 1}$ and Robin C. Jackson ${ }^{2}$ \\ ${ }^{1}$ Centre for Cognition and Neuroimaging, Brunel University, Uxbridge, UB8 3PH, \\ UK. ${ }^{2}$ Institute of Human Performance, University of Hong Kong, 111-113 Pokfulam \\ Road, Pokfulam, Hong Kong.
}

\begin{abstract}
.
Sporting performance makes special demands on perceptual skills, but the neural mechanisms underlying such performance are little understood. We address this issue, making use of fMRI to identify the brain areas activated in viewing and responding to video sequences of tennis players, filmed from the opponent's perspective. In a blockdesign, fMRI study, 9 novice tennis players watched video clips of tennis play. The main stimulus conditions were (1) serve sequences, (2) non-serve behaviour (ball bouncing) and (3) static control sequences. A button response was required indicating the direction of serve (left or right for serve sequences, middle button for non-serve and static sequences). By comparing responses to the three stimulus conditions, it was possible to identify two groups of brain regions responsive to different components of the task. Areas MT/MST and STS in the posterior part of the temporal lobe responded either to serve and to non-serve stimuli, relative to static controls. Serve sequences produced additional regions of activation in parietal lobe (bilateral IPL, right SPL) and in right frontal cortex (IFGd, IFGv), and these areas were not activated by non-serve sequences. These regions of parietal and frontal cortex have been implicated in a "mirror neuron" network in the human brain. It is concluded that the task of judgement of serve direction produces two different patterns of response: activations in MT/MST and STS concerned with primarily with the analysis of motion and body actions, and activations in parietal and frontal cortex associated specifically with the task of identification of direction of serve.
\end{abstract}

Keywords: Tennis; sport; observation of action; fMRI; mirror neurons.

Abbreviations: fMRI, functional magnetic resonance imaging; MT/MST middle temporal visual area, containing area V5 and other visual motion areas; STS, superior temporal sulcus; TMS, transcranial magnetic stimulation; RM representational momentum; AIP, anterior intraparietal sulcus, ROI, region of interest; SPL, superior parietal lobule; IPL inferior parietal lobule; IFGd, inferior frontal gyrus, dorsal region; IFGv, inferior frontal gyrus, ventral region;

*Corresponding author.

E-mail address michael.wright@,brunel.ac.uk 


\section{Introduction.}

The aim of the research presented here is to identify and analyse neural correlates of anticipatory skills in tennis players. However there have been few (if any) previous attempts to use functional imaging to analyse sporting performance and the majority of fMRI studies of perception and action have used carefully - controlled artificial stimuli. Therefore, as a first step in this challenging programme of work, we seek an identification of brain regions involved in processing and responding to the visual information associated with the viewing of tennis serves, and that is the purpose of the present paper. We will now briefly review some recent research that provides an insight into the possible neural mechanisms that give rise to perceptual performance in sport.

Analysis of the visual information contained in body movements begins in the striate occipital cortex of the brain, and proceeds to area V5 and adjacent visual motion areas in the posterior middle temporal gyrus (MT/MST), then to the superior temporal sulcus, STS (Grossman and Blake, 2002). It is known from the work of Johansson (1973) that viewing the motion of a small number of point-light sources attached to head, limbs and trunk gives an overwhelming impression of a human body engaged in recognisable movements, such as walking and running. Functional imaging experiments using point-light displays have implicated STS as important for the visual analysis of biological motion - the motion of human or animal bodies - based on purely kinetic cues (Grossman and Blake, 2002). This activation of STS is apparent not only relative to random motion controls (scrambled point-light displays), but also is greater than that to non-biological motions (of equivalent meaningfulness and complexity). STS responds even more strongly to videos of human motion than to point-light displays, suggesting integration of other visual cues with the basic kinetic information (Beauchamp, et al. 2003). Overall STS seems to be the most important region for the analysis of biological motion (Grèzes et al., 2001, Peuskens, et al. 2005).

In understanding the task of predicting the outcome a movement such as a tennis stroke, it is important to acknowledge that a kind of motion perception can occur even when there is no physical motion present. One example of this is the use of "frozen motion" pictures; a mid-motion snapshot of a moving object can contain implicit motion information, and fMRI studies (Kourtzi and Kanwisher, 2000; Senior et al., 2000) show that implied and actual motion share a common neural substrate in the MT/MST area. The task used by Senior et al (2000) enabled the activity of continuous and freeze-frame (implied) motion of the same objects to be compared, giving conclusive co-localisation of the regions of MT/MST activated by implied and actual motion. Another aspect of implicit motion is representational momentum (RM) where the remembered position of a moving object is displaced in the direction of motion, so that there is a kind of extrapolation effect from viewing a motion sequence (Freyd and Finke, 1984). There is good evidence that visual motion areas MT/MST are of primary importance in RM. Senior et al. (2002) showed that if the activity in the region of MT/MST is blocked by transcranial magnetic stimulation (TMS) then the RM effect in freeze-frame stimuli is also blocked, showing that MT/MST activity is necessary for the RM effect to occur. Conversely, different areas, in lateral occipital cortex, respond to static images of the human body and body parts (Downing, et al. 
2001) and these areas are unlikely to contribute to the task of anticipation of a body movement.

The analysis and interpretation of observed body actions is known to involve somatosensory (parietal lobe) and motor (frontal lobe) systems, as well as the visual motion and biological motion areas of the posterior temporal and adjacent occipital cortex. "Mirror neurons" in monkey frontal cortex discharge both when the monkey performs an action and when he observes a similar action performed by another monkey or by the experimenter (Rizolatti, et al. 1996). These bimodal neurons receive inputs from neurons of area AIP (anterior inferior parietal sulcus), thus forming part of a larger action/observation system (Jeannerod, et al. 1995). Functional imaging studies in humans have identified corresponding regions in frontal cortex and parietal cortex that are activated both by the perception of an action and the production of an action (Decety, et al., 1997; Hamzei, et al. 2003; Buccino, et al., 2004). One of the most significant outcomes from fMRI research is the identification of systems concerned with the perceptual analysis of actions, such as the purposeful and expressive movements of other humans. A revelation from this research has been the very close coupling of these perceptual systems with the action production systems of the brain.

On the basis of the fMRI studies of observed actions (reviewed above) we may formulate several specific hypotheses about the expected pattern of fMRI activations when viewing video sequences of tennis play. (1) Observation of serve sequences (relative to static control stimuli) will activate the posterior middle temporal gyrus (MT/MST visual motion area), and the STS biological motion area. (2) Activation of exactly these same areas, relative to static control stimuli, will also be produced by the observation of other movements of a tennis player that are unconnected with the delivery of a serve. (3) Active engagement of the observer in a task of predicting serve direction will induce stronger activation in these areas. (4) Active engagement on the task of predicting serve direction will also recruit a wider somatosensory and motor network. The purpose of the present study is thus to identify areas of the brain activated by observation of the spontaneous movement of a tennis player in between play, and to differentiate these from brain areas activated when the tennis player is observed specifically in order to predict the direction of a serve.

\section{Methods.}

\subsection{Stimuli}

The stimulus sequences were colour video clips in mpeg format, played at 25 frames/s, back projected and viewed in the fMRI scanner via an overhead mirror. The tennis player subtended approximately $4 \mathrm{deg}$, and between clips there were blank grey frames matched to the mean luminance of the tennis sequences. Each sequence was approximately $2 \mathrm{~s}$ in length, triggered by a scanner pulse, thus each sequence played once within every $4 \mathrm{~s}$. There were three principal types of stimulus, representing the three main experimental conditions: serve sequences, non-serve sequences and static control sequences. Non-serve motion sequences consisted of pre-serve behaviour (bouncing the ball with the racquet) matched in length to the serve sequences, and static control sequences showed a single static picture of the tennis player (from the ball bouncing clips) for the same length of time. 


\subsection{Participants}

Nine healthy adult novice tennis players (age $18-59 \mathrm{yr}$ ) with normal visual acuity took part in the study. Four were male and 5 were female. All were right-handed. Behavioural data were obtained for a further 20 participants (age $18-37 \mathrm{yr}$ ) outside the scanner in order to obtain reliable quantitative estimates of task performance.

\subsection{Design of the fMRI experiment}

The key issue is to identify different brain processes involved in the visual analysis of motion and action, on the one hand, and in the task of anticipation and judgement of direction, on the other hand. However, the exact balancing of experimental and control conditions is difficult with such naturalistic stimuli. We note that that the serve stimulus contains both a stronger motion stimulus (higher local image velocities) and a more vigorous body action stimulus than the non-serve condition. In turn, the non-serve condition entails a stronger motion stimulus than the static condition (we assume minimal implied motion in the static stimulus). Therefore, with respect to the visual motion and biological motion content of the stimuli we can write:

Serve $>$ non-serve $>$ static $=0$.

Also, the serve condition requires a difficult directional judgement, requiring purposeful attention to the body actions. Neither the non-serve nor the static condition requires any directional judgement at all. With respect to the judgment of direction, we can write:

Serve $>$ non-serve $=$ static $=0$

All three stimulus conditions do however require a simple identification of the type of sequence (serve, versus non-serve or static), and a button press. The three stimulus conditions are thus well balanced for these aspects of the task. Also, the three stimulus conditions are well balanced for their stationary visual content.

A block-design, fMRI study requires the creation of "contrasts" or comparisons, so that the significant differences in the BOLD response between experimental conditions can be determined. From our three experimental conditions, static, nonserve and serve, we derive three contrasts: non-serve - static, serve - non-serve and serve - static. From the analysis given above, we can make some predictions about the pattern of response we might expect to find in different brain areas to our three experimental contrasts.

For areas that analyse visual motion and/or biological motion:

Serve-static $>$ serve-non-serve,

and

Non-serve - static $>0$.

For areas that analyse directional judgement:

Serve - static $>$ non-serve - static $=0$, 
and

Serve - non-serve $>$ non-serve - static $=0$.

Analysis of all three contrasts will thus enable us to determine whether different brain areas respond differentially according to (a) analysis of visual motion and actions, and (b) directional judgement of action outcomes.

\subsection{Presentation schedule of stimuli.}

A block design was utilised for the fMRI experiment, with each block consisting of 5 sampled serve sequences of either of two tennis players, plus one $4 \mathrm{~s}$ blank slide, making a block length of $24 \mathrm{~s}$. Additionally there was a $24 \mathrm{~s}$ baseline (blank screen) condition that allowed periods of rest for the participant (and stabilisation of the BOLD response) at the beginning and end of the experiment and at two equally spaced intervals in the middle of the experiment. Serve sequences were terminated (temporal occlusion) either before or after the ball/racquet contact. Within a block, the same serve sequence was shown with 5 decreasing levels of temporal occlusion relative to the ball/racquet contact time $(-160,-80,0,+80,+160 \mathrm{~ms})$. Thus within a block it became progressively easier to judge the direction of the serve. The reason for adopting this progressive method of presentation was to maximise the attention and engagement of participants with the task. There were 9 blocks: two right-serve blocks, two left-serve blocks, two non-serve blocks, two static blocks, and one baseline (blank screen) block. These were presented four times each in a fixed pseudorandom order, making a total of $9 \times 4 \times 6(\times 1$ per TR $)=216$ brain volumes collected.

\subsection{Behavioural task}

The participants held a button box in their preferred hand while observing the stimuli in the scanner. They were instructed (verbally, and by on-screen instructions at the start of the scan) to give one of 3 finger presses for each video clip. If they thought that the serve was directed at the left court, they pressed the leftmost button. If they thought that the serve was directed at the right court, they pressed the rightmost button. If they thought that the sequence was not a tennis serve (i.e. Static and NonServe conditions) they pressed the centre button. They were instructed to anticipate the direction of the serve and make a forced-choice decision of left versus right for serve sequences, even when no part of the ball flight was visible. It was observed that performance of participants in the scanner was above $75 \%$ correct only in the last two trials of a block, when part of the ball flight was visible. There were no errors in identifying non-serves. This indicated that our fMRI observers were attentive to the task, but not whether their performance was typical for novice observers. The behavioural experiment was therefore repeated with a larger participant sample outside the scanner. Results are shown in Table 1 and showed chance performance if occlusion occurred before the ball/racquet contact, and near perfect performance if only one frame of the ball flight was visible. This behavioural task, as presented in the scanner, is widely used in the sports science literature, and results are entirely consistent with published data for novice observers (Farrow \& Abernethy, 2003; Jackson, 2003; Tennenbaum et al., 2000). 
Table 1.

Percentage correct responses of 20 novice observers in judging serve direction.

\begin{tabular}{|l|l|l|l|l|l|}
\hline$\%$ Correct & \multicolumn{5}{|l|}{ Temporal occlusion relative to ball-racquet contact } \\
\hline $\mathrm{N}=20$ & $-160 \mathrm{~ms}$ & $-80 \mathrm{~ms}$ & $0 \mathrm{~ms}$ & $80 \mathrm{~ms}$ & $160 \mathrm{~ms}$ \\
\hline Right serve & 47 & 51 & $73 *$ & $98^{* *}$ & $99 * *$ \\
\hline Left serve & 41 & 38 & 60 & $95 * *$ & $99 * *$ \\
\hline
\end{tabular}

$* \mathrm{p}<0.05, * * \mathrm{p}<0.005$, one-sample t-test with Bonferroni correction.

The parametric effect of the level of temporal occlusion on fMRI is not analysed here, but is the subject of a further study.

\section{6. fMRI data acquisition}

Imaging was performed with a Siemens Trio 3T MRI scanner using an 8-channel array head coil. For each functional run, an ultra fast echo planar gradient echo imaging sequence sensitive to blood-oxygenation-level-dependent (BOLD) contrast was used to acquire 32 slices per TR (3-mm thickness). The following parameters were used: $\mathrm{TR}=4000 \mathrm{~ms}, \mathrm{TE}=35 \mathrm{~ms}$, flip ANGLE $=90^{\circ} .216$ whole brain volumes were collected per participant. High resolution $(1 \times 1 \times 1 \mathrm{~mm})$, whole brain, T1-weighted structural images were also acquired using a standard 3D sequence.

\subsection{Data analysis}

fMRI data analysis was carried out using SPM2 http://www.fil.ion.ucl.ac.uk/spm. Functional images were realigned, co-registered with the T1 image, spatially normalised into standard space, re-sampled to $3 \times 3 \times 3 \mathrm{~mm}$ voxels and smoothed with a Gaussian kernel of $6 \mathrm{~mm}$ FWHM. The T1 images were also spatially normalised and resampled to $1 \times 1 \times 1 \mathrm{~mm}$ voxels. The fMRI time series in each voxel of the functional image was high pass filtered to $1 / 128 \mathrm{~Hz}$. First-level analysis involved estimation of the fixed effects of experimental conditions in a block design, according to the general linear model. This involved creation of contrasts between experimental conditions. The contrasts analysed were: (1) Non-Serve minus Static, (2) Serve minus Static and (3) Serve minus Non-Serve. In order to take into account the betweenparticipant variation, a Regions of Interest (ROI) analysis was then carried out using MarsBaR toolbox for SPM2 http://marsbar.sourceforge.net/. Analysis was conducted using a set of $10 \mathrm{~mm}$ radius spherical ROIs centred on locations identified from the first level analysis. The volume of the ROI was selected as a compromise between sensitivity (a smaller volume will deliver a potentially higher signal / noise ratio), and accuracy of location (there is a scatter in individual brain anatomy). The probable anatomical labels and the locations of the selected regions of interest in relation to anatomical landmarks were identified using the SPM2 version of the MSU space utility http://www.fil.ion.ucl.ac.uk/spm/ext/\#MSU and was confirmed by visualisation using the ICBM View tool available from the Montreal Neurological Institute, http://www.bic.mni.mcgill.ca/cgi/icbm_view/. The second level analysis consisted of analysis of variance carried out on the contrast values of individual participant brains within each ROI. 


\section{Results:}

\subsection{First level fMRI group analysis.}

This first-level analysis gives an overall, qualitative indication of results. The serve and non-serve sequences (measured relative to the static control) activate several brain areas in common. These common activations are shown in the left two columns of Figure 2. The MT/MST complex is strongly activated by both types of stimuli. Additionally, small foci of activation can be seen in the right parietal lobe and bilaterally in the frontal cortex.

We can also examine the differences the activations produced by viewing serve and non-serve sequences, and these differences are shown in the right-hand two columns of Figure 1. We note that the serve sequences produce additional activations, over and above that produced by non-serve sequences, in parietal and frontal cortex.

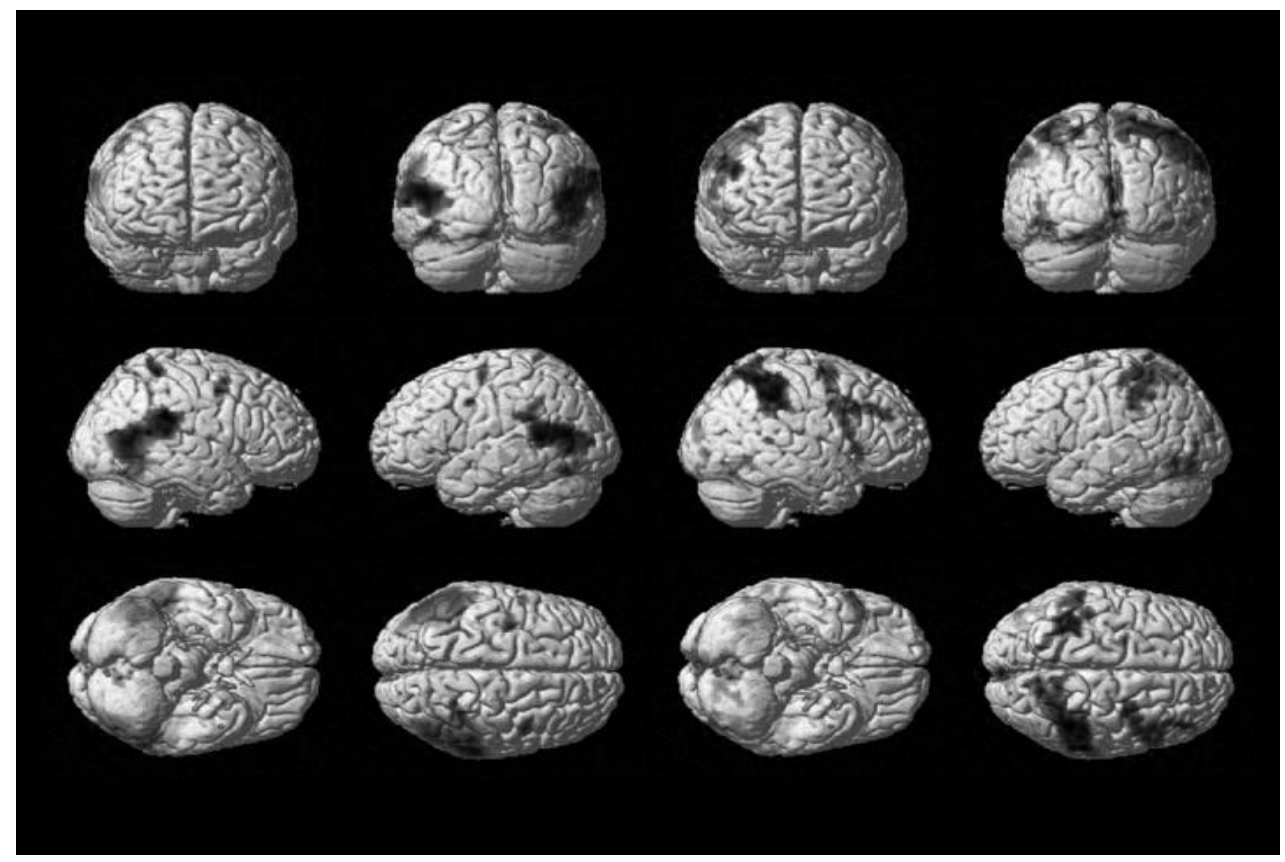

Figure 1. Columns 1 and 2 show the regions (dark shading) that were activated in common both by serve sequences and by non-serve sequences (as compared with static controls). Columns 3 and 4 show those regions of the brain where activation was exclusive to serve sequences, and where no activation was produced by nonserve sequences. The former are expected to reflect the general analysis of visual motion and action and the latter the determination of the direction of the serve. 


\subsection{Second level, regions of interest fMRI group analysis.}

To test the experimental hypotheses, taking into account individual variation, a second-level, regions-of -interest (ROI) analysis was carried out. Centres for regions of interest were identified from the local maxima in the first-level (fixed-effects) analysis. We were also interested in any possible hemispheric differences, so for paired regions of interest, we took average symmetrical centre co-ordinates in left and right hemispheres. The size of the ROIs (10 mm radius) was large in relation to any anatomical asymmetries between left and right hemisphere, and individual differences in sulcal patterns. The ROI centres were identified as located in posterior middle temporal gyrus (MT/MST) $( \pm 48-6712)$, superior temporal sulcus (STS) $( \pm 49-48$ $13)$, superior parietal lobule (SPL) $( \pm 29-6159)$, inferior parietal lobule (IPL) $( \pm 40-$ $4658)$ and both dorsal (IFGd) ( \pm 541222$)$ and ventral (IFGv) ( \pm 56240$)$ regions of inferior frontal gyrus.

The mean effect size (per voxel) was then determined in each ROI for each participant, for the three contrasts examined (non-serve - static, serve - non-serve, serve - static). The largest activations overall were obtained with the serve - static contrast in MT/MST L and MT/MST R.
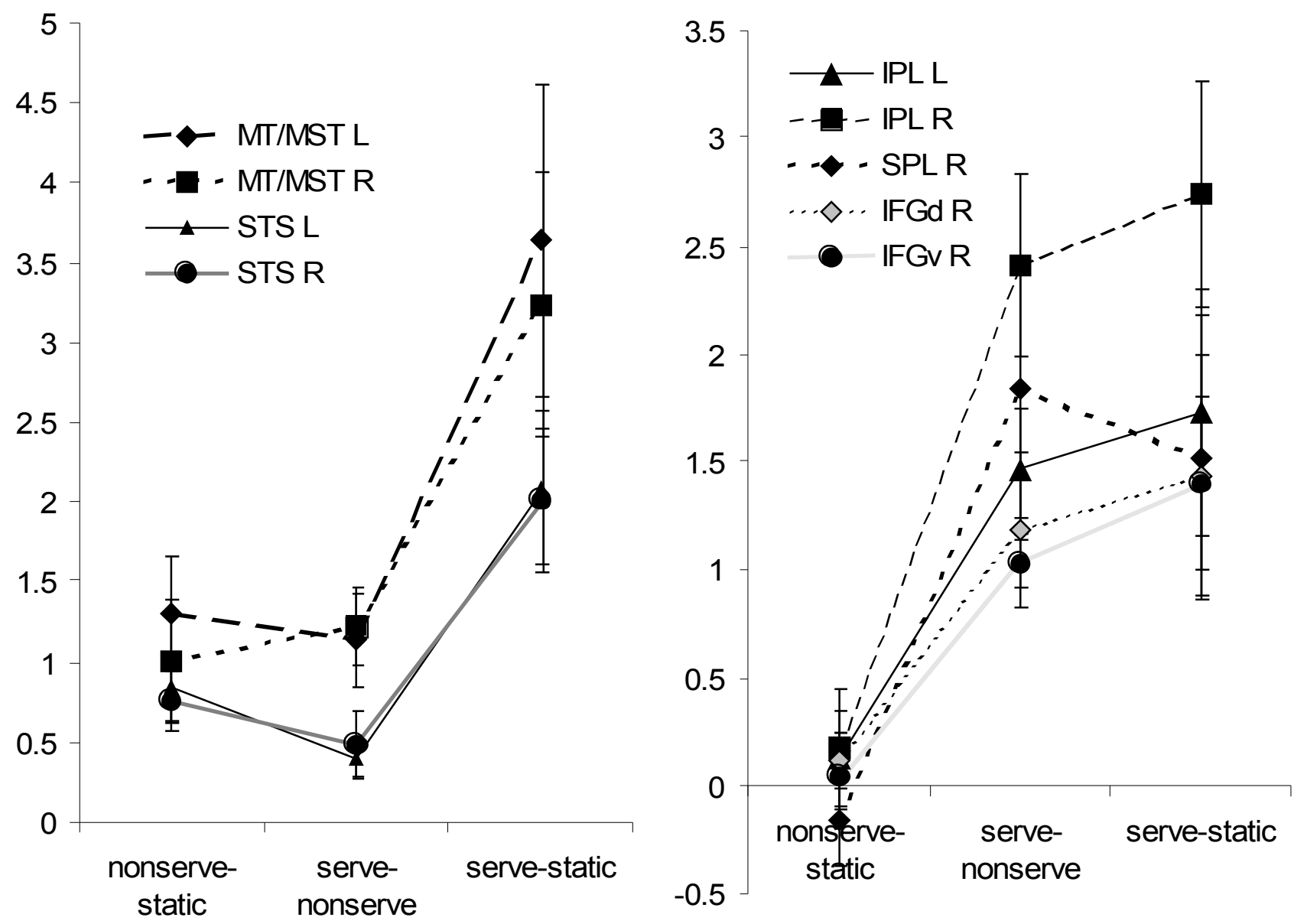
Figure 2. Distinct patterns of response to the experimental conditions occur in different regions of interest. In the left graph are regions showing a pattern characteristic of responsiveness to visual motion and body actions: left and right posterior middle temporal gyrus (MT/MST) ( $\pm 48-6712)$, and left and right superior temporal sulcus (STS) ( $\pm 49-48$ 13). The right graph shows data following a pattern characteristic of responsiveness to judgement of serve direction: right superior parietal lobule (SPL R) (29-61 59), left and right inferior parietal lobule (IPL) ( \pm 40 -4658 ) and right inferior frontal gyrus (dorsal 5412 36, ventral 56240 ). Vertical axis shows effect size \pm 1 s.e.m.

To test the statistical significance of the effects in relation to the experimental hypotheses, we conducted repeated measures analysis of variance. Three factors were analysed: experimental contrast (non-serve - static, serve - non-serve, serve - static) hemisphere (right, left) and region (MT/MST, STS, SPL, IPL, IFGd, IFGv). The hypothesis is that different brain regions respond differentially to the three experimental contrasts. This was confirmed: there was a significant interaction between experimental contrast and region $(\mathrm{F}=5.3$; $\mathrm{df}=3.3,26 ; \mathrm{p}<0.005$ with Greenhouse-Geissler correction). The 3-way interaction, experimental contrast $\mathrm{x}$ region $\mathrm{x}$ hemisphere was not significant.

Post-hoc planned comparisons (paired-samples t-tests) were carried out to determine whether the response to the three experimental contrasts, in each ROI, conformed to the "motion action pattern" or the "direction judgment pattern" (as defined in section 2.3. Design of the fMRI experiment). These patterns can be seen in the results presented in Figure 2, for which the statistical analysis is given below ${ }^{1}$. ROIs demonstrating a "motion action pattern" are shown in the left half of figure 3 . ROIs demonstrating a "direction judgement pattern" are shown in the right half of figure 3. The results of Figure 2 are consistent with the qualitative pattern of active areas shown in the fixed-effects analysis (Figure 1). The ROI analysis confirms statistically

\footnotetext{
${ }^{1}$ Let us call non-serve - static $=$ contrast $(a)$; serve - non-serve $=$ contrast $(b)$ and serve - static $=$ contrast (c). Applying paired sampled t-tests for selected comparisons, a "motion action pattern" (see Methods) predicts a significant difference between (a) and (c) and between (b) and (c) but not between (a) and (b), and also (by a one-sample t-test) that (a) is significantly greater than zero. Applying pairedsample t-tests to selected comparison, a "direction judgement pattern" predicts a significant difference between (a) and (c) and between (a) and (b) but not between (b) and (c). The "direction judgement pattern" (see Methods) also (by a one-sample t-test) predicts that (a) is not significantly different from zero. These patterns may be seen in the results presented in Figure 2, for which the statistical analysis is given below.
}

The following ROIs exhibited a "motion action pattern":

MT/MST L (c-a, t=3.7, p<0.01; c-b, t=3.0, p<0.05; a-b, n.s.; $a>0, t=3.7, p<0.01$ )

MT/MST R (c-a, t=4.7, p<.005; c-b, t=2.6, p<0.05; a-b, n.s.; $\mathrm{a}>0, \mathrm{t}=2.6, \mathrm{p}<0.05)$,

STS L (c-a t=4.5, p<0.005; c-b, $\mathrm{t}=3.8, \mathrm{p}<.01 ; \mathrm{a}-\mathrm{b}, \mathrm{n} . \mathrm{s}$.; $\mathrm{a}>0, \mathrm{t}=3.8, \mathrm{p}<0.01)$,

STS R (b-a t=4.2, c-a p<.005; c-b, t=3.9, p<0.005; a-b n.s.; $a>0, t=3.9, p<0.005)$.

The following ROIs exhibited a "direction judgement pattern":

IPL L (b-a, $\mathrm{t}=3.9, \mathrm{p}<0.005 ; \mathrm{c}-\mathrm{a}, \mathrm{t}=4.1, \mathrm{p}<0.005$; c-b n.s.; $\mathrm{a}>0$, n.s.),

SPL R (b-a, t=2.9, p<0.05; c-a, 3-1 t=2.8, p<0.05; c-b, n.s.; $a>0$, n.s.),

IPL R (b-a, $\mathrm{t}=3.7, \mathrm{p}<0.01 ; \mathrm{c}-\mathrm{a}, \mathrm{t}=6.7, \mathrm{p}<0.0005 ; \mathrm{c}-\mathrm{b}, \mathrm{n} . \mathrm{s} . ; \mathrm{a}>0$, n.s.),

IFGd R (b-a, $\mathrm{t}=4.1, \mathrm{p}=0.005 ; \mathrm{c}-\mathrm{a}, \mathrm{t}=6.3, \mathrm{p}<0.0005$; $\mathrm{c}-\mathrm{b}, \mathrm{n} . \mathrm{s}$.; $\mathrm{a}>0$, n.s.

IFGv R (b-a, t=2.5, p<0.05; c-a, t=2.7, p<0.05; c-b, n.s.; $a>0$, n.s.).

The following (left frontal lobe) ROIs exhibited no significant activations: IFGd L, IFGd L.

SPL L showed a trend similar to SPL R but did not meet all four statistical criteria. 
that there are two distinct sets of brain areas responding to tennis play sequences: a set of regions responding to the visual motion and body movement (MT/MST; STS) and a set of regions involved in the judgement of the direction of the serve (IPL L, IPL R, SPL R, IFGd R, IFGv R).

\section{Discussion.}

Observation of video clips of tennis serves produced strong activation of the brain's visual motion and biological motion areas, in comparison with observation of static frames from the same video clips. Likewise, observation of non-serve behaviour (ball bouncing) activated these same regions, relative to static controls. Strong localised clusters of activation were produced in the posterior middle temporal gyrus (MT/MST) and superior temporal sulcus (STS) in response to both serve- and nonserve video sequences and these activations were stronger to serve stimuli than to non-serve stimuli. Additionally, a cluster of activations was produced in parietal and right frontal cortex by the serve sequences but not by the non-serve sequences (in relation to static control stimuli). The additional areas form part of the "mirror network" associated with the viewing and production of actions. The involvement of frontal and parietal areas in the response to serve sequences cannot be attributed simply to the motor response, since a button press was required in all conditions. Nor can it be attributed simply to observation of an action, since the activation in these areas produced by viewing the non-serve action was minimal. Activations seen in parietal and frontal lobe regions of interest showed a qualitatively different pattern across experimental conditions from those seen in MT/MST and STS. We attribute the activation in these areas specifically to the task of judging serve direction.

We shall now consider the identification of the ROI locations in the current study in relation to findings reported in the literature. The comparison of localisation data across published studies is at best approximate (Brett, et al., 2002) and to minimise error, we restrict ourselves to fMRI studies that have published co-ordinates normalised to the same MNI template as used in the present study. The criterion for agreement is that the centres of activation in the listed studies falls within the $10 \mathrm{~mm}$ radius ROI as defined in the present study. It is possible to identify separate clusters of activation in our experiments both in regions responding to a wide range of motion stimuli (MT/MST) and in areas that respond specifically to biological motion stimuli (STS) (Grezes, et al., 2001). The greater activation of MT/MST by the serve stimulus relative to the non-serve stimulus could be due to stimulus differences, such as the distribution of image velocities, or to greater attention to motion (Buchel, et al. 1998); likewise the greater activation of STS could be due to greater attention to biological motion (Hirai, et al. 2005). There was stronger activation of these areas from serve sequences than from non-serve sequences. Additionally, a cluster of cortical areas in parietal and right frontal lobes was activated strongly by the serve stimuli, in comparison with either non-serve stimuli or static stimuli. The pattern of response to the three experimental contrasts was similar for parietal and frontal areas, and differed from that in MT/MST and STS. The region that we have identified as inferior parietal lobule, IPL, overlaps both the classical "mirror neuron" area of intraparietal sulcus, and a more posterior area, thought to be involved in the visual analysis of motor actions (Grezes, et al., 2003; Hamzei, et al., 2003; Buccino, et al. 2004). The SPL region is less often associated with the "mirror network" but Buccino et al. (2004) found that it was activated by observation of actions that subsequently required a 
related motor response (as in the present study). Finally, two regions within the inferior frontal gyrus, IFGd and IFGv, showed a pattern of activation that linked them specifically with the judgment of serve direction. These regions have been associated with the "mirror network": the first of these specifically with the preparation of action (Buccino, et al. 2004) and the second with the recognition of actions (Hamzei et al., 2003; Buccino, et al., 2004).

There have been other recent fMRI studies of naturalistic actions, confirming that the "mirror system" is involved in certain complex forms of motor performance that require imitation. Calvo-Merino et al. (2005) studied professional ballet dancers, professional capoeira dancers and non-expert controls. Greater activation was found in premotor cortex, intraparietal sulcus, right superior parietal lobe and left posterior temporal sulcus when expert dancers viewed their own style of dance relative to the other style. Similar effects of expertise may be predicted for sport. Buccino, et al. (2004) found that the "mirror system" was involved in learned imitative performance, in an fMRI study involving the learning of guitar chords by musically naïve participants. Activation of the "mirror system" is not limited to contexts involving imitation of movement: it is also found in the preparation of purposeful actions such as grasping an object, independent of actual execution of a movement (Grezes, et al. 2003).

Another perceptual process that may contribute to expertise in sporting performance is the estimation of time-to-contact (TTC). Field and Wann (2005) examined fMRI activations in a task that required judgements of TTC of an expanding optic flow pattern. Activations were identified in superior parietal and in motor cortex, and these could not be attributed to any overt movements of the participants. They concluded that the networks involved in extracting TTC from optic flow are in close correspondence with the sensory and motor systems that would be involved in generating a relevant critically timed action such as catching or hitting a ball or avoiding collision. A further testable possibility is a role for implicit motion or representational momentum (RM) in the task of prediction of the direction of a tennis serve. If RM contributes to the performance in such a task, this would underline a possible adaptive function for RM in enhancing the predictive power of motion perception. The current behavioural data however suggest that any representational momentum from frames prior to the ball-racquet contact is insufficient to provide accurate prediction of the serve direction, at least for novice observers.

Sports such as tennis, cricket and badminton make strong demands on perceptual systems and reaction time, and the ability to make anticipatory responses on the basis of early cues is critical to expertise (Farrow and Abernethy, 2003). For example, Glenross and Cibich (1977) calculated that a cricket ball bowled at $40.2 \mathrm{~ms}^{-1}$ took 439 ms to reach the batsman. In this time, the batsman must recognise the type of delivery and respond appropriately. Even allowing for a relatively short movement time of 250 $\mathrm{ms}$ and a response latency of $200 \mathrm{~ms}$, it is evident that a decision must be based, at least in part, upon information gleaned prior to the bowler releasing the ball (see also McLeod, 1987). Similar calculations in tennis suggest it is not possible to make an accurate return to a serve based solely upon analysis of ball-flight characteristics. There have been extensive studies of anticipatory skills in sport, in which the visual information available to an observer is cut off at some precise time before or after a key event: this is known as "temporal occlusion". The key event for tennis and other 
racquet sports is normally the ball/racquet contact. Both video-based studies in the laboratory with manual responses, and studies on the tennis court with natural wholebody responses (using liquid crystal goggles to vary the perceptual information available) have shown that experts are better able to anticipate than novices. Prediction of ball direction by experts and novices has been studied (in squash) both with film displays or matched point-light displays (Abernethy, et al. 2000). Earlier pick-up of visual information was found for experts in the point-light task, just as was is found the film task, suggesting that the superior performance of the experts is directly related to their superior processing of kinematic information. Spatial occlusion studies (in badminton) have shown that the movement of the arm and racquet is the source of the critical cues for racquet sports (Abernethy, 1991). These techniques may soon be adaptable to provide an analytical approach to functional imaging studies of the perception and analysis of actions using ecologically valid tasks. How can these time-critical processes be studied with fMRI, whose temporal resolution is limited? We are currently analysing the fMRI activations produced in the tennis task by different amounts of temporal occlusion in the judgement of serve direction. Some further progress may be achieved using event-related fMRI. The event-related design employed by Buccino et al. (2004) (in their study of imitation of guitar chords) allowed them to track the successive involvement of different neural structures in the observation of an action, and the subsequent preparation and execution of a response.

It is concluded that in novice tennis players observing a tennis serve, visual motion areas of MT/MST and STS are strongly stimulated, and these areas are also activated by observation of the spontaneous between-play motions of the player. Additionally, there is a differential involvement of specific regions of the parietal and right frontal cortex for engagement in the task of prediction of serve direction, which respond only weakly to the general observation of spontaneous actions.

Acknowledgement. We thank Adrian Williams for comments on an earlier draft of this paper.

\section{References:}

Abernethy, B., 1990. Expertise, visual search, and information pick-up in squash. Perception, 19, 63-77.

Abernethy, B., 1991. Anticipation in squash: differences in advance cue utilisation between expert and novice players. Journal of Sports Sciences, 8, 17-34.

Abernethy, B., Gill, D.P., Parks, S.L. \& Packer, S.T., 2001. Expertise and the perception of kinematic and situational probability information. Perception, 30, $233-$ 252.

Beauchamp, M.S., Lee, K.E., Haxby, J.V., \& Martin, A., 2003. fMRI responses to video and point-light displays of moving humans and manipulable objects Journal of Cognitive Neuroscience 15, 991-1001

Brett, M., Johnsrude, I.S. \& Owen, A.M., 2002. The problem of functional localisation in the human brain. Nature Reviews Neuroscience, 3, 243-249. 
Buccino, G., Vogt, S., Ritzi, A., Fink, G.R., Zilles, K., Freund, H-J. \& Rizzolatti, G., 2004. Neural circuits underlying imitation learning of hand actions: an event related fMRI study. Neuron, 42, 323-334.

Buchel, C., Josephs, O., Rees, G., Turner, R., Frith, C. D., and Friston, K. J. 1998. The functional anatomy of attention to visual motion. A functional MRI study. Brain $121,1281-1294$.

Calvo-Merino, B., Glaer, D.E., Grèzes, J., Passingham, R.E. \& Haggard, P., 2005. Action observation and acquired motor skills: an fMRI study with expert dancers. Cerebral Cortex, 15, 1243-1249.

Downing, P. E., Jiang, Y., Shuman, M., \& Kanwisher, N., 2001. A cortical area selective for visual processing of the human body. Science, 293, 2470-2473

Farrow, D. \& Abernethy, B., 2003. Do expertise and the degree of perception - action coupling affect natural anticipatory performance? Perception, 32, 1127-1139.

Farrow, D., Abernethy, B. \& Jackson, R.C., 2005. Probing expert anticipation with the temporal occlusion paradigm: experimental investigations of some methodological issues. Motor Control, 9, 330-349.

Field, D.T. \& Wann, J.P., 2005. Perceiving time to collision activates sensorimotor cortex. Current Biology (In Press).

Freyd, J.J. \& Finke, R.A., 1984. Representational momentum. Journal of Experimental Psychology: Learning, Memory and Cognition, 10, 126-132.

Glencross, D. J., \& Cibich, B. J., 1977. A decision analysis of games skills. Australian Journal of Sports Medicine, 9, 72-75.

Grèzes, J., Fonlupt, P., Bertenthal, B., Delon-Martin, C., Segebarth, C. \& Decety, J., 2001. Does perception of biological motion rely on specific brain regions?

NeuroImage, 13, 775-785.

Grèzes, J., Armony, J.L., Rowe, J \& Passingham, R.E., 2003. Activations to "mirror" and "canonical" neurons in the human brain: an fMRI study. NeuroImage, 18, 928937.

Grossman, E. \& Blake, R., 2002. Brain areas active during visual perception of biological motion. Neuron, 35, 1167-1175.

Hamzei, F., Rijntjes, M., Dettmers, C., Glauche, V., Weiller, C. \& Buchel, C., 2003. The human action recognition system and its relationship to Broca's area: an fMRI study. Neuroimage, 19, 637-644.

Hirai, M., Senju, A., Fukushima, H. \& Hiraki, K., 2005. Active processing of biological motion perception: an ERP study. Cognitive Brain research, 23, 387-396. 
Jackson, R.C., 2003. Evaluating the evidence for implicit perceptual learning: a reanalysis of Farrow and Abernethy. Journal of Sports Sciences, 20, 471-485.

Jackson, R. C., \& Farrow, D., 2005. Implicit perceptual training: How, when, and why? Human Movement Science, 24, 308-325.

Jeannerod, M., Arbib, M.A., Rizzolatti, G. \& Sakata, H., 1995. Grasping objects: the cortical mechanisms of visuomotor transformation. Trends in Neuroscience, 18, 314320.

Johansson, G., 1973. Visual perception of biological motion and a model for its analysis. Perception and Psychophysics, 14, 201-211.

Kourtzi, Z. \& Kanwisher, N., 2000. Activation in human MT/MST by static images with implied motion. Journal of Cognitive Neuroscience, 12, 48-55.

McLeod, P., 1987. Visual reaction time and high-speed ball games. Perception, 16, 49-59.

Peuskens, H., Vanrie, J., Verfaillie, K., \& Orban, G.A., 2005. Specificity of regions processing biological motion. European Journal of Neuroscience, 21, 2864-2875.

Rizzolatti, G., Fadiga, L., Gallese, V. \& Fogassi, L., 1996. Premotor cortex and the recognition of motor actions. Cognitive Brain Research, 3, 131-141.

Senior, C., Barnes, J., Giampetro, V., Simmons, A., Bullmore, E.T., Brammer, M. \& David, A.S., 2000. The functional neuroanatomy of implicit-motion perception or "representational momentum". Current Biology, 10, 16-22.

Senior, C., Ward, J. \& David, A.S. (2002). Representational momentum and the brain: an investigation into the functional necessity of V5/MT. Visual Cognition, 9, 81-92.

Tennenbaum, G., Sar-El., T., \& Bar-Eli, M., 2000. Anticipation in ball location in low and high-skill performers. A developmental perspective. Psychology of Sport and Exercise, 1, 117-128. 